\title{
2D Hexagonal Covalent Organic Radical Frameworks as Tuneable Correlated Electron Systems
}

\author{
Raul Santiago ${ }^{1}$, Isaac Alcón ${ }^{2}$, Jordi Ribas-Arino ${ }^{1}$, Mercè Deumal ${ }^{1}$, Ibério P. R. Moreira ${ }^{1}$, and
} Stefan T. Bromley ${ }^{1,3, *}$

${ }^{1}$ Departament de Ciència de Materials i Química Física \& Institut de Química Teòrica i Computatcional (IQTCUB), Universitat de Barcelona, c/ Martí i Franquès 1-11, 08028 Barcelona, Spain

${ }^{2}$ Physical and Theoretical Chemistry Department, Freie Universität Berlin, Arnimallee 22, 14195 Berlin, Germany

${ }^{3}$ Institució Catalana de Recerca i Estudis Avançats (ICREA), Passeig Lluis Companys 23, 08010 Barcelona, Spain

*Corresponding author: s.bromley@ub.edu

Quantum materials hold huge technological promise but challenge our fundamental understanding of complex electronic interactions in solids. The Mott metal-insulator transition on half-filled lattices is an archetypal demonstration of how quantum states can be driven by electronic correlation. Twisted bilayers of 2D materials provide an experimentally accessible means to probe such transitions, but these seemingly simple systems belie high complexity due to the myriad of possible interactions. Herein, we show that electron correlation can be simply tuned in experimentally viable 2D hexagonally ordered covalent organic radical frameworks (2D hex-CORFs) based on single layers of half-filled stable radical nodes. Our carefully procured theoretical analysis predicts that can be varied between a correlated antiferromagnetic Mott insulator state and a semimetallic state via means of modest out-of-plane compression. This work establishes 2D hex-CORFs as a class of versatile single layer quantum materials to advance our understanding of complex low dimensional electronic systems. 


\section{Introduction}

Hexagonally ordered single layers (SL) of three-fold connected $\mathrm{sp}^{2}$ carbon sites ( $\alpha \mathrm{C}$ centres) possess one singly occupied non-hybridized $2 p$ orbital per site. For weak inter-site interactions these $2 p$ electrons tend to form a gapless semimetallic state with Dirac-like linear dispersive bands, as exemplified by SL graphene. ${ }^{1}$ The ability to tune the electron-electron coupling in graphene would permit access to correlated gapped electronic states which could open the door to further technological applications. Theoretical work has suggested that application of high $(>15 \%)$ in-plane biaxial strain to SL graphene could induce a transition from semimetallicity to a correlated antiferromagnetic Mott insulating (AFMI) state. ${ }^{2,3}$ More recent calculations, however, show that an AFMI phase in strained graphene would likely always be less stable than competing diamagnetic insulating valence bond (or quinoidal) states which start to emerge at $<15 \%$ strains, ${ }^{4}$ and that are also likely to play a precursor role in structural failure ${ }^{5}$. Unlike in-plane strain, out-of-plane compression has recently proven to be viable tool by which to tune the electronic correlation in bilayer (BL) graphene. Specifically, a recently observed correlated insulating state in twisted BL graphene $^{6}$ was shown to be dynamically tuneable by applying up to 2.1 GPa out-of-plane compressive stress $^{7}$ to induce transitions to new states (e.g. superconducting, magnetic). Although the underlying physics is not yet fully understood, the tunability of twisted BL hexagonal systems offers hope that analogous behaviour could be found in simpler, potentially easier to analyse, SL systems. Herein, we show how correlated electronic states could be finely tuned in experimentally viable hexagonal SL carbon-based systems via relatively very modest $(<0.05 \mathrm{GPa})$ out-of-plane compression.

Recently, we proposed a new class of $\mathrm{sp}^{2}$ carbon-based SL materials based on linking trivalent triarylmethyl (TAM) aC-containing radical molecules to make two dimensional (2D) covalent organic radical frameworks (CORFs). ${ }^{8,9}$ Since our prediction, some example 2D-CORFs have been experimentally prepared..$^{10,11,12}$ of particular interest in the present work are 2D CORFs with hexagonal connectivity based on the graphene-like honeycomb lattice (2D hex-CORFs), which we predicted to exhibit spin-polarised antiferromagnetic (AF) ground states and low energy valence bond quinoidal gapped states, linked by an intervening semimetallic transition state. ${ }^{9}$ Compared to the delocalised semimetallic state the more stable AF and valence bond states have lower space group symmetries and thus can be described as emerging through symmetry breaking. ${ }^{9,13}$ The first experimental examples of a 2D hex-CORF were indeed found to exhibit an AF ground state ${ }^{10,11} 2 \mathrm{D}$ hex-CORFs can be seen as expanded graphene lattices where chemical linkers, rather than external strain, increase the distance between the three-fold connected carbon sites. In this respect 2D hex- 
CORFs are similar the hypothetical $\alpha$-graphyne ${ }^{14}$ and $\alpha$-graphdiyne ${ }^{15}$ where the relatively increased distance between $\mathrm{sp}^{2}$ carbon nodes leads to a reduction in the inter-node electronic coupling (i.e. renormalized $t$ values). Unlike these hypothetical planar carbon allotropes, 2D hex-CORFs are based on sterically protected $\mathrm{sp}^{2}$ carbon nodes (e.g. TAMs) leading more experimentally realisable materials. TAMs are stable and versatile open-shell species which exhibit the Kondo effect in molecular junctions, ${ }^{16}$ and have been employed as building blocks for molecular spintronics, ${ }^{17}$ nonvolatile memory platforms, ${ }^{18}$ and nanoporous magnets. ${ }^{19}$ The experimentally synthesised TAM building blocks we consider are also not planar, but exhibit twisted aryl rings whose twist angle largely determined their electronic properties. ${ }^{20}$ In turn, SL TAM-based 2D hex-CORFs exhibit out-ofplane atoms and thus have a small finite thickness. In this computational work, we show that the twist angles of aryl ring linkers in 2D hex-CORFs are highly sensitive to applied out-of-plane compression, which can thus be used to modulate the inter-node electronic coupling and thus tune the electrical, magnetic and optical responses. Specifically, we show that out-of-plane compressive stress could enable the realisation of an AFMI-to-semimetallic transition in suitably fabricated 2D hex-CORFs, thus highlighting the potential importance of this class of SL materials for studying correlated electronic states.

Our theoretical exploration of 2D hex-CORFs upon compression has been carried out by means of all-electron density functional theory (DFT) based calculations and a mapping of the DFT results onto a Hubbard-like model Hamiltonian. Previously we have confirmed that the interlinked electronic and structural properties of individual TAMs can be well captured by DFT-based calculations. ${ }^{20}$ Building on previous work, ${ }^{8,9}$ we further confirm that the properties of TAM-based 2D hex-CORFs can be well captured by suitable DFT calculations, and thereby show how their electronic states can be tuned by compression. DFT is a formally exact method ${ }^{21}$ to determine the electron density of quantum systems without the need for an explicit many body treatment. In practical implementations, DFT-based calculations depend on a description of electron correlation through an approximate exchange-correlation potential $\left(V_{x c}\right)$. To improve the accuracy of DFT-based treatments of the electronic structure of correlated systems, it is well established that $V_{x c}$ can be significantly improved through incorporation of exact non-local Hartree-Fock exchange (HFE). The resulting hybrid DFT-based methods have become very effective approaches to investigate the electronic structure of different types of systems, ranging through strongly correlated transition metal oxides, $^{22,23,24}$ organic radicals ${ }^{25}$ and polyradicals, ${ }^{26,27,28}$ with a remarkable reliability in describing their ground states and magnetic properties. Hybrid functionals provide a computationally effective approach that can be applied in periodic schemes, and which give a good description of magnetic properties for many classes of compounds. Although quantitative results can be sensitive to the 
exact percentage of HFE employed, most hybrid functionals use between $20-50 \%$ HFE and tend to provide qualitatively similar results. Our DFT-based calculations employ $25 \%$ HFE through use of the PBEO functional ${ }^{29}$, to provide a self-consistent description of all electrons in our studied systems.

The use of model Hamiltonians to describe interactions between only those electrons in the system which are thought to be most important is a complementary approach to DFT. The half-filled (i.e. one electron per site) Hubbard model on the honeycomb lattice ${ }^{30}$ can thus be used to describe the essential electronic structure of SL hexagonal frameworks of $\mathrm{sp}^{2}$ carbon sites. This model describes the interplay between: i) a localising effective short-range electrostatic repulsion between electrons on single sites $(U)$, and ii) a kinetic energy term $(t)$ which describes the capacity of electrons to move between sites. Due to the formal simplicity of such models, they are more amenable to being solved through accurate many body methods. Such approaches have revealed that the ground state phase diagram of this model exhibits graphene-like semimetallicity for smaller $U / t$ ratios, and a continuous quantum transition to a correlated AFMI state when $U / t$ is increased. ${ }^{31,32}$ Generally, the Hubbard model is a useful generic model for the essential physics of half-filled 2D systems, but extensions to the model are likely to be necessary for more precise descriptions of specific experimental systems. For example, extended Hubbard-like models with both short- and long-range components of the electron-electron repulsion ${ }^{33}$ can improve the description of different physical realisations of SL half-filled 2D Dirac fermionic systems on the honeycomb lattice (e.g. graphene on different substrates, artificial graphene made from quantum corrals $^{34}$ ). However, the presence of heteroatoms or different kinds of hybridised sites (such as in 2D hex-CORFs) leads to highly complex models that are only solvable approximately using mean field or limited correlated schemes.

In this work, we make a link between the DFT-based and model Hamiltonian approaches by extracting approximate $U$ and $t$ values from our PBEO calculations. In the extreme case of a molecular crystalline lattice of isolated (i.e. non-linked) TAMs, $t$ is extremely small and $U$ is high leading to a highly robust AFMI state that has been experimentally shown to be resistant to pressures of over $20 \mathrm{GPa} .{ }^{35}$ In Ref. 36 relatively high $\mathrm{U} / \mathrm{t}$ ratios $(6.3-16.0)$ were obtained from DFT calculations of a few unstrained 2D hex-CORF structures. According to the Hubbard model for the 2D honeycomb lattice, these linked structures also have ground states that are firmly within the AFMI part of the phase diagram (i.e. for $U / t>3.8$ )..$^{31,32}$ This result is in line with the calculated ${ }^{9,36}$ and experimentally observed ${ }^{10}$ stability of AF phases reported for unstrained $2 D$ hex-CORFs. Due to the approximations in both DFT and model Hamiltonian approaches outlined above, one should be careful not to overinterpret the physical significance of theoretically derived $t$ and $U$ values with respect to any specific material. However, the consistency between the stable states predicted by 
DFT-based calculations and the Hubbard model phase diagram for high $U / t$ ratios indicate that taking both descriptions into account could be useful for exploring the electronic structure of 2D hex-CORFs with lower $U / t$ ratios. Following the experimentally demonstrated compression-induced tunability of correlated states in twisted BL graphene ${ }^{7}$, we show that application of out-of-plane compression to $2 \mathrm{D}$ hex-CORFs can dramatically reduce their $U / t$ values. We further note that unlike typical magnitudes of out-of-plane compressive pressures used to show observable effects in experiments on other 2D materials (e.g. 1- 2.5 GPa for graphene ${ }^{7,37}$, 0.5-5 GPa for SL $\mathrm{MoS}_{2}{ }^{38,39}$ ), we predict that relatively small compressions $(<0.05 \mathrm{GPa})$ would be needed for $2 \mathrm{D}$ hex-CORFs. Specifically, we show that such modest out-of-plane compression should enable an AFMI-tosemimetallic transition in suitably fabricated 2D hex-CORFs, thus highlighting the potential importance of this class of SL materials for studying 2D correlated electronic systems.

\section{Results}

We consider three examples of TAM-based 2D hex-CORFs based on: a) ring-sharing (rs) triphenylmethyl (TPM) radicals ${ }^{9}$ - TPM $_{\mathrm{rs}}$ b) ring-sharing perchlorotriphenylmethyl (PTM) radicals ${ }^{9}$ PTM $_{r s}$, and c) acetylenic linked PTM radicals as found in a recently synthesised and experimentally characterised material (PTM acetylenic), ${ }_{1}^{10,11}$ see Figure 1. We note that although extended 2D hex-CORFs a) and b) have not yet been synthesised, recently, it has been experimentally demonstrated that one can connect TPMs via ring-sharing in molecules of containing a ring of six $\alpha \mathrm{C}$ centres (i.e. a TPMrs fragment). ${ }^{40}$ This synthetic breakthrough opens the door to the possibility of extended $2 \mathrm{D}$ hexCORFs based on ring-sharing TAMs, such our proposed PTM $r$ and TPM-based materials. The structures of all three 2D hex-CORFs are shown in figure 1. 


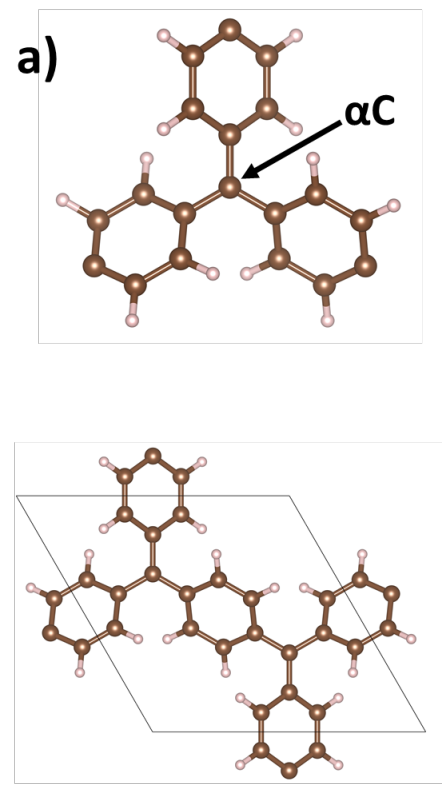

TPMrs

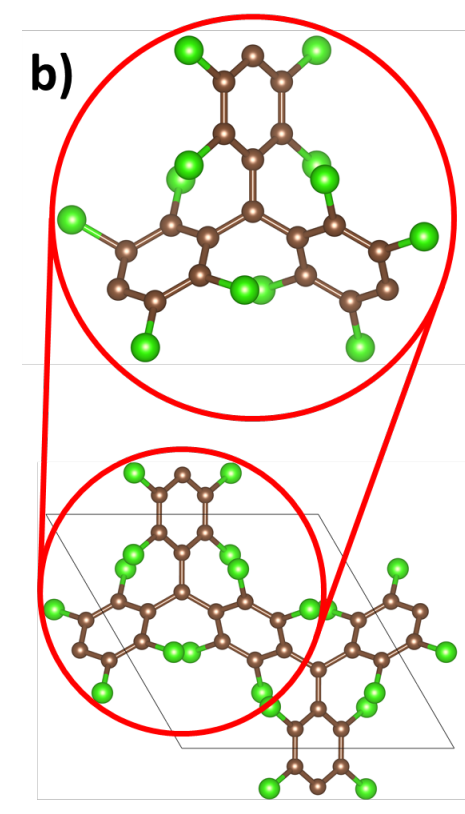

PTMrs
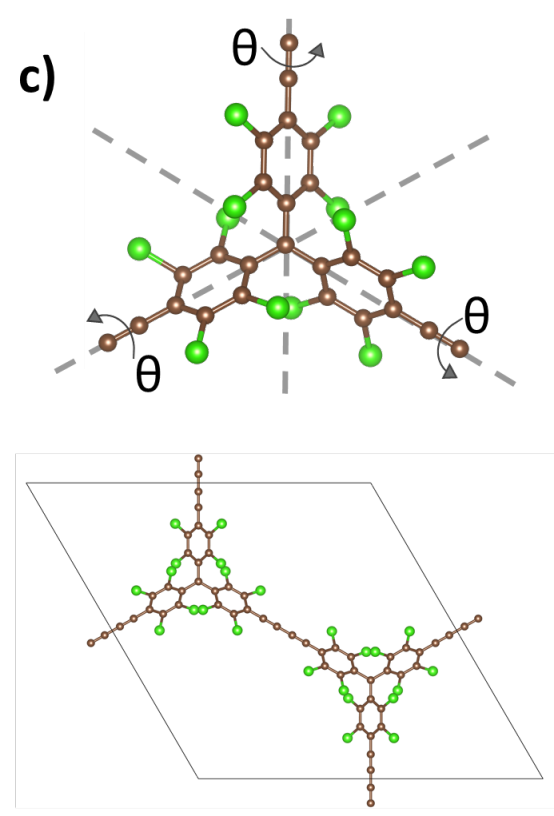

PTMacetylenic

Figure 1. Structures of the three considered 2D hex-CORFs: a) ring-sharing triphenylmethyl $\left(T_{P M} M_{\mathrm{rs}}\right)$ radicals, b) ring-sharing perchlorotriphenylmethyl $\left(\right.$ PTM $\left._{\mathrm{rs}}\right)$ radicals, and c) acetylenic coupled PTM radicals (PTM acetylenic $_{1}$. Lower: cuts from each extended 2D hex-CORF structure with the unit cell delimited by a grey line. Upper: local chemical environment of the corresponding protected triconnected $\mathrm{sp}^{2}$ carbon sites $(\alpha C)$ for each structure. In $c$ ) we highlight the aryl ring dihedral angles $(\theta)$ that are affected by compression in all structures. Atom colour key: carbon - dark brown, hydrogen - light brown, chlorine - green.

We note that, at variance with purely planar SL systems such as graphene, out-of-plane hydrostatic compression significantly affects the dihedral angles and bond distances in 2D hexCORFs. These changes govern electron localisation in the hexagonal lattice of $\alpha \mathrm{C}$ centres in these materials, which remains planar throughout. Other materials with non-planar layers (e.g. black phosphorous) have attracted interest due to their electronic response upon compression. ${ }^{41}$ In black phosphorous, compression affects the coordinates of all atoms in the puckered layers which affect band dispersion/ordering, which, in turn, can cause a semiconductor-metal transition at $1.2 \mathrm{GPa}$. Unlike in 2D hex-CORFs this is not a correlated electronic response. For each of our considered 2D hex-CORFs we first optimise the cell parameters and all atomic positions of each system to obtain an unstrained reference point. Using an approach established in other studies modelling compressed SL materials, ${ }^{39,42}$ we apply compressive stress to each 2D hex-CORF by employing selected out-of-plane (i.e. $z$-direction) structural constraints while allowing for full relaxation of the $2 \mathrm{D}$ lattice and unconstrained atoms (see Methods section and SI for details). With respect to the original z- 
direction thickness we apply compression to each system such that each $2 \mathrm{D}$ hex-CORF is compressed by a maximum of $60 \%$ (i.e. to $40 \%$ of the original thickness). For each material we have calculated the perpendicular (i.e. in a direction normal to the plane of the layer) ${ }^{43}$ Young's modulus (see SI for details) thus enabling us to provide an estimate of the required out-of-plane pressure to induce any percentage of compression. We find that the perpendicular Young's moduli (TPM $\mathrm{rs}_{\mathrm{rs}}$ : $0.085 \mathrm{GPa}$, PTM $_{\mathrm{rs}}$ : $0.41 \mathrm{GPa}$, PTM acetylenic: $0.046 \mathrm{GPa}$ ) imply that very modest pressures (i.e. $<0.05 \mathrm{GPa}$ ) are needed to obtain the reported compressions.

In figures $2 a-b$ the main compression-induced structural responses are shown for each 2D hex-CORF. Upon applying increasing compression to the unstrained materials, the dihedral angles of the aryl rings with respect to the $\alpha \mathrm{C}$ centres decrease (i.e. ring flattening) in a linear manner, in line with the applied constraints. The main effect of ring flattening is to increase $\pi-\pi$ orbital overlap between the between $\alpha \mathrm{C}$ centres and nearest neighbour carbon $(\mathrm{nnC})$ atoms. The increased $\pi-\pi$ overlap also leads to an associated increase in spin delocalisation ${ }^{20,8}$ which we show for PTM $_{\text {rs }}$ in an FM state in figure $2 c$. The in-plane bond $\alpha \mathrm{C}$-nnC bond distances show more complicated non-linear dependence on out-of-plane compression, although in all cases tend to increase (i.e. bond stretching) for higher compressions due to increasing steric hindrance between aryl rings. In summary, during out-of-plane compression, there is a competition between increased $\alpha \mathrm{C}$-nnC bond strength due to ring flattening and associated increased $\pi-\pi$ overlap, and increasing steric hindrance between aryl rings which tends to expand the in-plane $\alpha \mathrm{C}-\mathrm{nnC}$ bonds

In $\mathrm{TPM}_{\mathrm{rs}}$ the initial average aryl ring dihedral angle $\langle\theta\rangle$ is the lowest of all considered materials $\left(\sim 32.6^{\circ}\right)$ due to the relatively low steric hindrance between the $\mathrm{H}$-functionalised aryl rings. ${ }^{20}$ At the maximum considered compression, ring flattening leads to a $\langle\theta\rangle$ value of only $12.9^{\circ}$. The in-plane $\alpha \mathrm{C}$-nnC bond lengths in TPM $\mathrm{T}_{\mathrm{rs}}$ start at just below $1.44 \AA$. This value is slightly higher than $\mathrm{C}-\mathrm{C}$ bond length in graphene (1.42 $\AA$ ), in line with the conjugated $\mathrm{C}-\mathrm{C}$ bonding throughout the $\mathrm{TPM}_{\mathrm{rs}}$ network. With increasing compression this bond length increases monotonically with a gradually increasing rate up to $1.458 \AA$ at $60 \%$ compression.

For $\mathrm{PTM}_{\mathrm{rs}}$ the more sterically hindered $\mathrm{Cl}$-functionalised aryl rings induce an initial $<\theta>$ value of $45.7^{\circ}$ which reduces to $17.5^{\circ}$ at maximum compression. PTM $\mathrm{PT}_{\mathrm{r}}$ has the same topologically connected carbon network but with higher $\langle\theta\rangle$ values that reduce the $\pi-\pi$ overlap, leading to longer average $\alpha \mathrm{C}-\mathrm{nnC}$ bond lengths than in TPM $\mathrm{M}_{\mathrm{rs}}$. However, unlike $\mathrm{TPM}_{\mathrm{rs}}$, for $0-25 \%$ compression the $\alpha \mathrm{C}$ $\mathrm{nnC}$ distances first decrease and then increase with increasing aryl ring flattening. This nonmonotonic behaviour can be understood due to the relatively reduced $\pi-\pi$ overlap in the longer relatively strained $\alpha \mathrm{C}-\mathrm{nnC}$ bonds in uncompressed PTM $\mathrm{rs}$. Unlike in uncompressed TPM $_{\mathrm{rs}}$ where the conjugation is relatively high and the initial $\alpha \mathrm{C}-\mathrm{nnC}$ bonds are quite short, in $\mathrm{PTM}_{\mathrm{rs}}$ ring flattening 
significantly increases the $\pi-\pi$ overlap leading to a strengthening and shortening of these bonds. For PTM ${ }_{\text {acetylenic, }}$ the aryl rings are also $\mathrm{Cl}$-functionalised and the initial $\langle\theta\rangle$ value $\left(48.2^{\circ}\right)$ is thus very similar to that of PTMrs. However, the $\pi$-conjugation through the longer acetylenic linkers is relatively lower and the initial average $\alpha \mathrm{C}-\mathrm{nnC}$ bond lengths are considerably longer (1.468 Å). Like PTM $_{\mathrm{rs}}$, applying compression also leads to an initial increase in $\pi-\pi$ overlap and a corresponding dip in the average $\alpha \mathrm{C}$-nnC distances. At higher compression PTMacetylenic also shows significant fluctuations in the average $\alpha \mathrm{C}-\mathrm{nnC}$ distances. These $\mathrm{C}-\mathrm{C}$ bond distance fluctuations are observed throughout the conjugated in-plane bonded carbon network of this material (see SI) and are thus likely to be linked to variations in $\pi-\pi$ overlap. We note that energetically competing spiral conjugated $\pi-\pi$ overlap possibilities have been reported in acetylenic chains ${ }^{44}$ which would sensitively depend on $\langle\theta\rangle$. We also note that these small bond length fluctuations does not appear to be an indication that the material is failing structurally, as can be confirmed through the small effect they have on the near-harmonic energy versus compression relation, implying elastic behaviour for this compression range (see $\mathrm{SI}$ ). 
a)
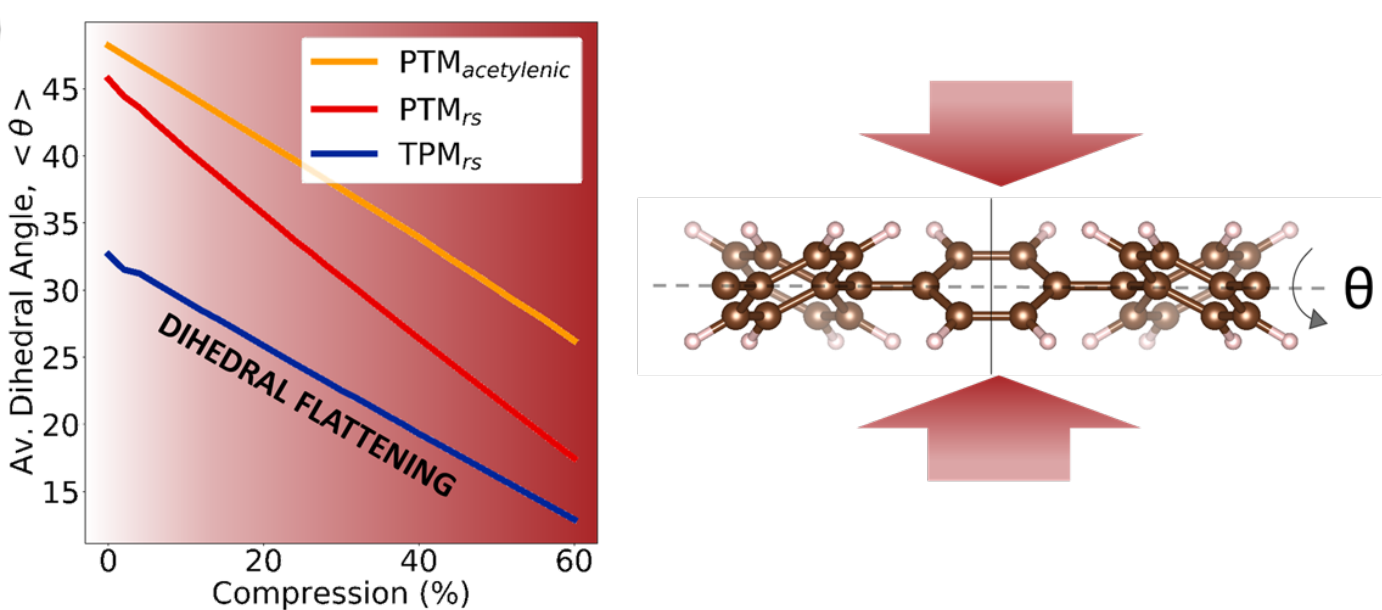

b)

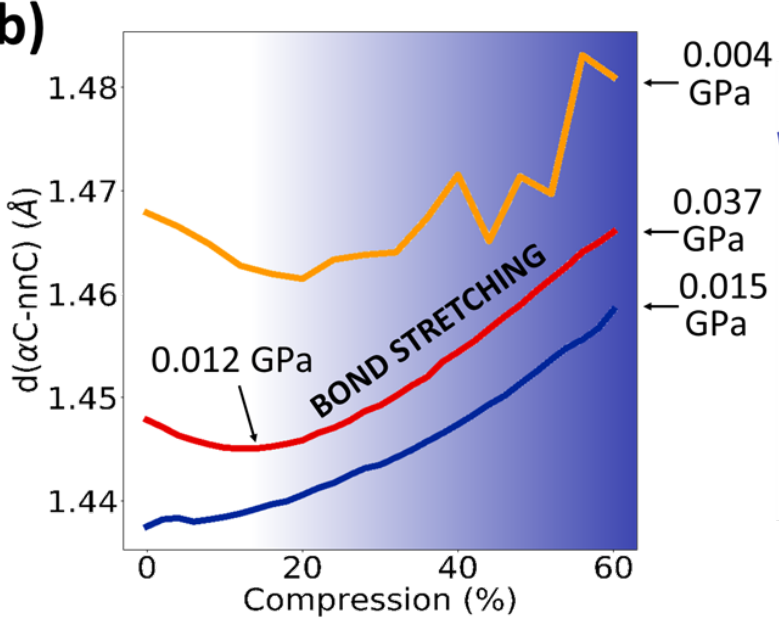

c)
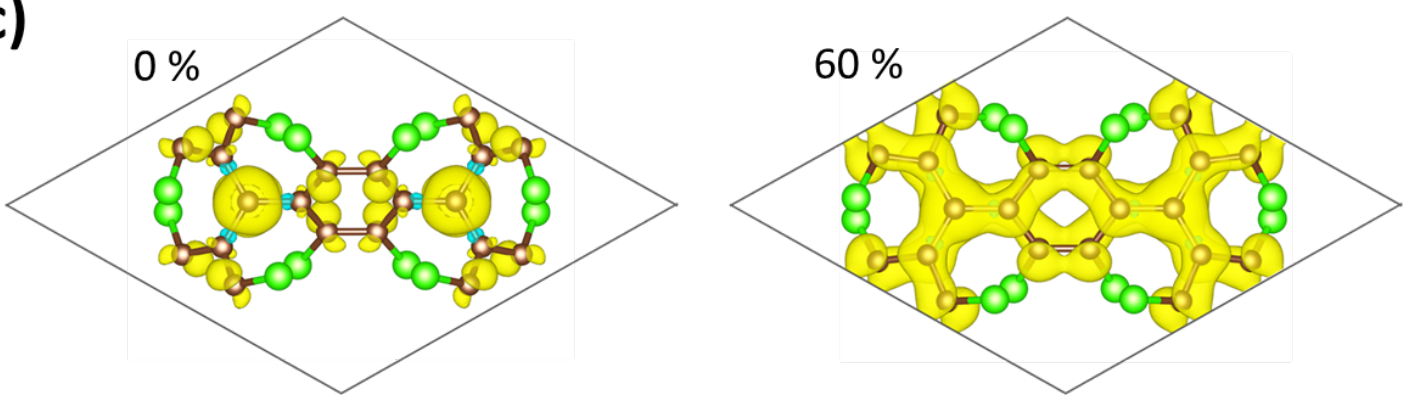

Figure 2. Response of 2D hex-CORFs upon compression. The contributions of ring flattening and inplane bond stretching in the AF state are indicated by plots of: a) average ring dihedral angle $\langle\theta\rangle$ (see Fig. 1), and b) average $\alpha C-n n C$ bond lengths (highlighted in red, right), respectively. The pressures in GPa required for selected compressions in each 2D hex-CORF are indicated in b). c) spin density (yellow/cyan) over the bonded C network of PTM $\mathrm{P}_{\mathrm{rs}}$ for $0 \%$ (left) and $60 \%$ (right) compression for the FM state.

The structural changes arising from compression induce a significant electronic response in the three 2D hex-CORFs. In all cases and for all considered compressions our DFT calculations predict a gapped AF ground state. However, with increasing compression for all 2D hex-CORFs the AF state is 
progressively energetically destabilised with respect to the semimetallic state (see Fig. 3a). This tendency can also be measured by the average absolute spin moment per $\alpha \mathrm{C}$, the AF coupling constant $(J)$ and the electronic band gap $(\Delta \mathrm{E})$, see Methods section and $\mathrm{SI}$ for details. In all three 2D hex-CORFs we find that these three indicators decrease with increasing compression (see Figs. 3b-d).

With respect to the parameters plotted in Fig. 3a-d, TPM $\mathrm{rs}_{\mathrm{s}}$ shows the mildest changes of all three 2D materials with respect to compression. Although TPM $_{r s}$ is the closest energetically to being semimetallic for most of the compression range, with correspondingly smaller spin densities and a relatively small AF band gap, the AF magnetic coupling in $\mathrm{TPM}_{\mathrm{rs}}$ is the largest of all considered

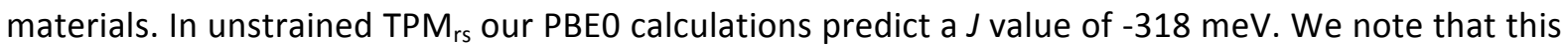
value appears to be fairly robust with respect to the percent of HFE used in the functional (see table S2). At $60 \%$ compression $J$ reaches $-386 \mathrm{meV}$ (see also Table 1 for selected $J$ values for each $2 \mathrm{D}$ hexCORF). Recently, a singlet-triplet energy gap of $248 \mathrm{meV}$ was measured for a synthesised molecular fragment of the TPM $\mathrm{rs}_{\mathrm{rs}}$ structure containing six spin $1 / 2 \alpha C$ sites in a ring. Using the exact solution for an $\mathrm{AF}$ hexagonal Heisenberg system of $S=1 / 2$ spin particles in a 6 -membered ring, in which $\left(E_{S}-E_{T}\right)=$ $1.369 J,{ }^{45}$ we obtain $J=-197 \mathrm{meV}$ for this experimental system. We note that the average dihedral angle in this molecule is $\sim 7$ degrees higher than in our extended TPM $_{\mathrm{rs}}$ material. In previous work we showed that the average dihedral angle of aryl rings in TAM-based 2D CORFs determines the energy difference between AFMI and FM states ${ }^{8}$. In order to compare with experimental, we linearly extrapolate the $J$ versus compression curve for TPM $_{r s}$ (to approximately $-20 \%$ compression), to better match the average dihedral angle value of the TPM-based ring molecule. In line with experiment, we find $J \approx-200 \mathrm{meV}$ for TPM $\mathrm{TP}_{\mathrm{rs}}$ under these conditions providing support for the suitability of our hybrid DFT approach for treating these systems.

For PTMacetylenic the AF state shows the largest energy difference with respect to the semimetallic state for all considered compressions, but with relatively weak AF coupling. For unstrained PTMacetylenic, we obtain $J=-15 \mathrm{meV}$. We note that our PTMacetylenic model is an infinite symmetric SL with an unpaired spin at every $\alpha \mathrm{C}$ site. However, experimental PTMacetylenic samples consist of a few finite-sized layers, each with a proportion of non-spin carrying sites and each likely to exhibit some structural defects. These differences may begin to explain why the fitting of experimental susceptibility data leads to a relatively larger $J$ value $(-46.5 \mathrm{meV})$ than we obtain. ${ }^{10}$ For this system, the highest considered compression destabilises the dominant AF state by $18.6 \mathrm{meV}$ per $\alpha \mathrm{C}$ with a concomitant reduction of the band gap by $0.28 \mathrm{eV}$. Recently, it was proposed that by replacing the PTM $\mathrm{rs}_{\mathrm{rs}}$ nodes in the PTM acetylenic structure with oxo-bridged planar TAMs, one could increase the calculated AF coupling strength by approximately four times. ${ }^{36}$ We note that oxobridged planar TAMs are relatively unstable to reaction with oxygen, ${ }^{46}$ unlike TAMs which are 
kinetically stabilised by sterically hindering access to the central $\alpha \mathrm{C}$ (e.g. PTMs and TPMs). As such, 2D hex-CORFs based on oxo-bridged planar TAMs are unlikely to be amenable to experimental synthesis. Herein, however, we predict that application of small compressive pressures $(0-0.05$ $\mathrm{GPa}$ ) to the stable as-synthesised PTMacetylenic structure one could tune the magnetic coupling and thereby achieve a $\times 2.5$ increase in AF coupling strength $(J=-37 \mathrm{meV})$ together with a significant drop $(>0.4 \mathrm{eV}$ ) in the electronic band gap (see Fig. 3). For PTM acetylenic at high compression we observe some fluctuations in all the measured electronic parameters (Figs 3a-d), which is also observed in the in-plane C-C bond distances (see Fig 2 and SI). As noted above, these variations are likely linked to different $\theta$-dependent $\pi$ - $\pi$ overlap possibilities in the acetylenic bridges.

Of the three considered 2D hex-CORFs, PTM $\mathrm{rs}_{\mathrm{rs}}$ shows the most dramatic electronic response with respect to compression. For no strain, our calculations predict PTM $\mathrm{P}_{\mathrm{rs}}$ to exhibit an AF state with a slightly larger energy gap than PTM acetylenic. With increasing strain, the PTMrs energy gap rapidly decreases to eventually be lower than that of both TPM $\mathrm{T}_{\mathrm{rs}}$ and $\mathrm{PTM}_{\text {acetylenic }}$ for $>50 \%$ compression. Moreover, for this high compression, PTM rs has the lowest spin density per atom and energetically is the closest to being semimetallic. As for TPM, the AF coupling is significant in this material, with $J=-$ $365 \mathrm{meV}$ for the highest compression. The evolution of the electronic band structure of PTM $\mathrm{rs}_{\mathrm{rs}}$ with increasing compression is shown in Fig. 3e, where the decrease in the energy gap and increase in overall band dispersion is clearly seen. For high compression the difference in energy between the $\mathrm{AF}$ and the semimetallic state for both $\mathrm{TPM}_{\mathrm{rs}}$ and $\mathrm{PTM}_{\mathrm{rs}}$ is calculated to be only $\sim 30 \mathrm{meV}$ per $\alpha \mathrm{C}$. Such marginal energy differences between distinct electronic states should be treated with caution within a single determinantal picture such as DFT. These results suggest that compression of $2 \mathrm{D}$ hexCORFs can make the semimetallic and AF states almost degenerate in energy, and both would contribute to the true ground state. 

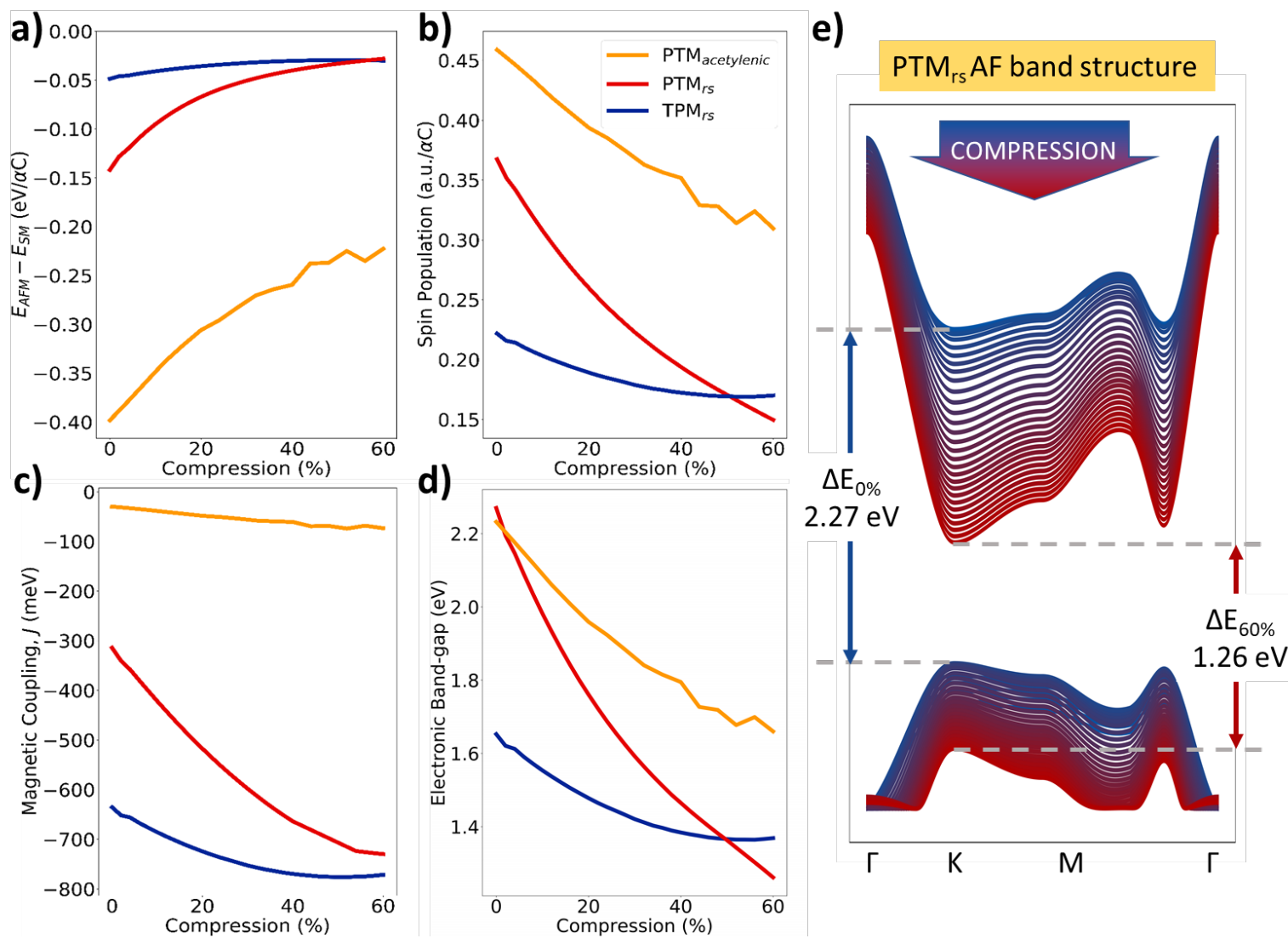

Figure 3. Directly extracted results from DFT-based calculations for out-of-plane compressions from 0 - 60\% for each 2D hex-CORF: a) energy difference between the AF and semimetallic state (eV per $\alpha C), b)$ average absolute spin moment per $\alpha C$ site, c) AF coupling constant, $J(m e V)$, d) AF electronic band gap (eV), e) evolution of the AF band structure of $\mathrm{PTM}_{\mathrm{rs}}$ from $0 \%$ (blue) to $60 \%$ (red) compression.

In addition to directly inspecting the nature of the lowest energy state predicted by DFT calculations, we can also extract $U$ and $t$ parameters from our calculations (see Methods section and SI for details) which we can use to compare with predictions from the Hubbard model on the honeycomb lattice. In figure 4 we plot the extracted values of $t, U$ and $U / t$ for all three 2D hex-CORFs for all considered degrees of compression (see also Table 1 for selected values). For TPMrs and PTM $M_{\text {acetylenic, }}$ compressing the unstrained structures up to $60 \%$ leads to a decrease in $U$ of $0.28 \mathrm{eV}$ and $0.57 \mathrm{eV}$ respectively. For both these 2D hex-CORFs this compression also leads to a similar increase in $t(0.3-0.5 \mathrm{eV})$. For PTM acetylenic the extracted $U$ values are relatively high compared to the $t$ values and this entails a corresponding increased sensitivity of the $U / t$ values which vary from 12.8 to 7.4. In ref. 24 , the smallest reported $U / t$ ratio of 6.3 was obtained in a proposed structure based on oxobridged planar-TAMs with vinylenic linkers. In both cases, these $U / t$ ratios firmly place these 
materials in the AFMI regime according to the Hubbard model phase diagram. For PTMacetylenic this picture is fully consistent with a direct inspection of our DFT results (see Fig. 3). Ideally, for exploring transitions between the AFMI and semimetallic phases in half-filled 2D-hexagonally ordered systems, one should be able to tuneably access the regimes above and below $U / t \approx 3.8^{31,32}$

For TPM, the $t$ values are relatively larger than the $U$ values and the corresponding $U / t$ ratio does not change significantly with compression (from $2.75-2.15$ ), which places TPM $_{\mathrm{rs}}$ in the semimetallic region of the Hubbard phase diagram. Direct inspection of our DFT results for TPM $\mathrm{rs}_{\mathrm{r}}$ indicates that the semimetallic state is always within $48.5 \mathrm{meV}$ per $\alpha \mathrm{C}$ of the AF state and that this energy difference slightly decreases with compression. Although, the quantitative picture provided by these two perspectives differs somewhat, both suggest that TPMrs has a ground state that is quite close to the boundary between AFMI and semimetallic phases and which is only slightly affected by compression.

PTM $_{\mathrm{rs}}$, unlike PTM acetylenic and TPM, shows a large and non-monotonic change in $t$ with respect to increasing compression. The $t$ values for PTM $\mathrm{P}_{\mathrm{rs}}$ lay between those extracted for TPM $\mathrm{M}_{\mathrm{rs}}$ and PTM $M_{\text {acetylenic }}$ but first increase by $0.04 \mathrm{eV}$ with a $0-10 \%$ compression increase and thereafter decrease by $0.26 \mathrm{eV}$ as compression increases to $60 \%$. This non-monotonic behaviour in $t$ is related to the analogous behaviour in the $\alpha \mathrm{C}$-nnC bond distances (see Fig. 2) which are a structural indicator of the electronic coupling between $\alpha C$ centres. The value of $U$ for uncompressed PTM $_{\mathrm{rs}}$ is higher than the corresponding value for PTMacetylenic but rapidly decreases with increasing compression and becomes lower than that of $\mathrm{TPM}_{\mathrm{rs}}$ for $60 \%$ compression. Correspondingly, the $U / t$ ratio for uncompressed PTM $\mathrm{r}_{\mathrm{rs}}$ starts at 4.52 , then decreases to a minimum value of 3.50 at $20 \%$ compression, and then gradually increases back up to 4.53 at the highest compression. From a Hubbard model perspective, the PTM ${ }_{r s}$ would have an AFMI ground state which could be tuned to be arbitrarily close to the AFMI-semimetallic phase transition boundary with applied compression above and below $20 \%$. A direct inspection of the DFT calculations also suggests that PTM Ps $_{\text {s }}$ exhibits an AF state that can be tuned to be energetically close to the semimetallic phase. However, the latter picture indicates a monotonic progression towards the semimetallic phase with increasing compression. These different tendencies are to be expected to due to differences in the methodologies employed. The electronic states in the Hubbard model are derived from a subset of the actual electrons on a fixed lattice, and effects such as polarisation/delocalisation of non- $\alpha \mathrm{C}$-based electrons and structural relaxations (e.g of the linkers) which are described by DFT calculations, are inherently not included. On the other hand, Monte Carlo treatments of the Hubbard model Hamiltonian explicitly describe electronic correlation and attempts to solve it using mean field based approaches (e.g. pure HF) tend to yield lower effective $U$ values than direct many body solutions. ${ }^{30}$ Hybrid DFT functionals attempt 
to partially bridge the gap between these approaches but results can be sensitive to the percent of HFE employed. In figure $4 \mathrm{c}$ we include shading to indicate tentative variations in $U / t$ with respect to modest changes in HFE percent.

\begin{tabular}{|c|c|c|c|c|c|c|c|c|c|c|c|c|}
\hline & \multicolumn{3}{|c|}{$J(\mathrm{meV})$} & \multicolumn{3}{|c|}{$U(e V)$} & \multicolumn{3}{|c|}{$t(\mathrm{eV})$} & \multicolumn{3}{|c|}{$U / t$} \\
\hline & $0 \%$ & $20 \%$ & $60 \%$ & $0 \%$ & $20 \%$ & $60 \%$ & $0 \%$ & $20 \%$ & $60 \%$ & $0 \%$ & $20 \%$ & $60 \%$ \\
\hline PTM ${ }_{\text {acetylenic }}$ & -15 & -2 & -3 & 2.23 & 1.96 & 1.66 & 0.17 & 0.20 & 0.22 & 12.8 & 9.63 & 7.39 \\
\hline PTM $_{\text {rs }}$ & -157 & -259 & -365 & 2.27 & 1.76 & 1.26 & 0.50 & 0.50 & 0.28 & 4.52 & 3.50 & 4.53 \\
\hline TPM $_{\mathrm{rs}}$ & -318 & -362 & -386 & 1.65 & 1.48 & 1.37 & 0.60 & 0.62 & 0.64 & 2.75 & 2.37 & 2.15 \\
\hline
\end{tabular}

Table 1. Extracted values of $J, U, t$ and $U / t$ for $\operatorname{PTM}_{\text {acetylenic, }} \operatorname{TPM}_{\mathrm{rs}}$ and $\mathrm{PTM}_{\mathrm{rs}}$ for selected compressions. The $U / t$ values are shaded according to the corresponding ground state on the Hubbard model phase diagram for the 2D half-filled hexagonal lattice: AFMI - orange, semimetallic blue.

\section{a)}

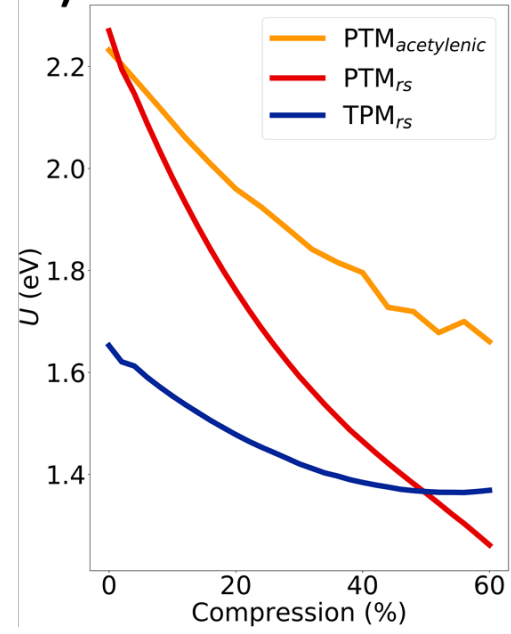

b)

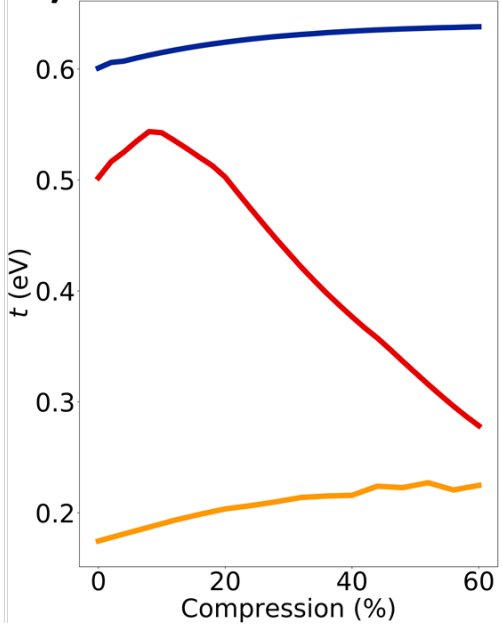

c)

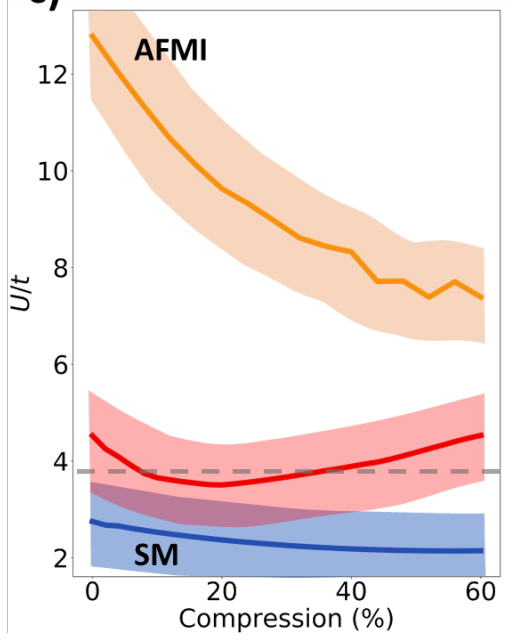

Figure 4. Extracted values of: a) $U$, b) $t$, and c) $U / t$ from our DFT calculations for PTMacetylenic, PTM rs and TPM $_{\mathrm{rs}} 2 \mathrm{D}$ hex-CORFs for a $0-60 \%$ range of compression. Dashed grey line in $\mathrm{c}$ ) indicates the boundary between AFMI and semimetallic (SM) phase stability $(U / t=3.8)^{31,32}$ with respect to the Hubbard model phase diagram for the 2D half-filled hexagonal lattice. The shading around the lines in c) provides a estimate of the expected variation in $U / t$ values with respect to modest changes in the percent of HFE used in the functional (see table S2).

\section{Conclusions}


$2 \mathrm{D}$ hex-CORFs are novel examples of the half-filled 2D honeycomb lattice and thus lie in the same class as graphene and hexagonal quantum corrals. ${ }^{33}$ Theoretical studies using simple but exactly solvable many body model Hamiltonians are still revealing new fundamental physical insights regarding the AFMI-semimetallic transition in such systems. ${ }^{31,32}$ However, even for these chemically uncomplicated systems, such simplified generic descriptions of can fail to capture essential features found in experiment. ${ }^{33} 2 \mathrm{D}$ hex-CORFs are relatively chemically complex materials that have a highly non-trivial structural and electronic response to out-of-plane compression. To accurately describe such complexity using a many body Hamiltonian approach would likely require a hugely complicated and intractable model. Here, we use an all-electron hybrid DFT approach to describe the full chemical structure and electronic response of our 2D hex-CORFs, while also extracting parameters relevant to generic model Hamiltonian descriptions (i.e $U, t, J$ ). The complementary picture thus obtained shows that compressing suitable $2 \mathrm{D}$ hex-CORFs could provide a new means for probing the phase diagram of this important 2D correlated electron system. Specifically, we demonstrate that TAM-based 2D hex-CORFs, like twisted BL graphene, are correlated electronic materials with states that can be tuned by out-of-plane compression. The twistable aryl rings in the SL materials we consider mean that only very modest out-of-plane pressures $(<0.05 \mathrm{GPa})$ are required to vary their electronic structure. This general result can be readily tested as we predict that very modest out-ofplane pressure applied to the experimentally synthesised PTMacetylenic material should lead to a significant increase in its observed AF magnetic coupling strength. Further, based on synthesised $T_{P M}$ molecular fragments, we propose that ring-sharing 2D hex-CORFs should be experimentally viable materials and would be particularly promising as they can: i) exhibit ground states laying close to the AFMI-semimetallic transition, and ii) have compression-tuneable $U / t$ ratios for low out-ofplane pressures. 2D hex-CORFs are thus promising materials, both from a fundamental point of view to study correlation effects in low dimensional materials and for potential technological applications (e.g. sensors, spintronics/electronics). We hope that our work will inspire further experimental and theoretical work into this fascinating new class of 2D correlated electronic materials.

\section{Methods}

Unstrained structures of each 2D hex-CORF were obtained via DFT-based optimisations of atomic positions and cell parameters using the $\mathrm{PBEO}^{29}$ hybrid functional as implemented in the all-electron FHI-AIMS code. ${ }^{47}$ In all calculations non-bonded dispersion interactions were included via the TSvdW approach. ${ }^{48} \mathrm{~A}$ "light/Tier1" numerical atom-centred orbital (NAO) basis set was used throughout. ${ }^{49}$ This basis set provides results of a similar or higher quality to those obtained with valence triple-zeta plus polarization Gaussian type orbitals ${ }^{50}$ in line with the trends regarding the quality of these NAO basis sets. ${ }^{51}$ The $2 \mathrm{D}$ hex-CORF SLs were oriented so that the $\alpha \mathrm{C}$ plane was 
parallel to the $x-y$ plane (with $z=0$ for the $\alpha C$ plane) and were separated from their periodic images by a vacuum spacing of $40 \AA$ in the $z$-direction. $6 \times 6 \times 1$ Monkhorst-Pack generated grid meshes of $k$ points were used for each 2D hex-CORF.

We use the constrained atom method ${ }^{39,42}$ to induce a $z$-directed out-of-plane compression on the 2D hex-CORFs. In ref. 39 the results from employing this method are shown to compare very well with experimental results from uniaxial out-of-plane compression on $S L \operatorname{MoS}_{2}$. For this we first select a set of $C$ atoms both above and below the $\alpha \mathrm{C}$ plane for each structure (see $\mathrm{SI}$ for specific atom sets in each case). To apply compression, the absolute value of the $z$-coordinates of these constrained atoms was adjusted according to incremental decreases of the dihedral angles of the aryl rings, and then fixed for each increment. The resulting $z$-constrained structures were then optimised again using the above set-up allowing for full relaxation of the unit cell, positions of nonconstrained atoms, and the $x$ and $y$ coordinates of the $z$-constrained atoms. In the SI we show that this constrained atom method produces the same structural response as when a $2 \mathrm{D}$ hex-CORF is sandwiched within a compressed layered 2D heterostructure. The perpendicular Young's moduli, and thus the external out-of-plane pressure required to induce these compressions, were extracted from the total energy versus unit cell volume curves (see SI for details). From these curves we could verify that our 2D hex-CORFs were always in the elastic regime for all considered compressions.

The magnetic coupling constants, J, were evaluated assuming the Heisenberg Hamiltonian whereby the energy difference between AFMI and FM states is directly related to the Heisenberg spin exchange parameter $J_{i j}$ (see $\mathrm{SI}$ for further details). The $t$ and $U$ values were extracted from the electronic band structures of each 2D hex-CORF following the procedure detailed in ref. 52 (see also SI).

\section{Acknowledgements}

This work was supported by the following research grants: MICIUN/FEDER RTI2018-095460-B-I00 and CTQ2017-87773-P/AEI/FEDER (Spanish government, MINECO), MDM-2017-0767 (“María de Maeztu" program for Spanish Structures of Excellence), 2017SGR13 and 2017SGR348 (Generalitat de Catalunya, DURSI). R.S. acknowledges funding from MINECO under grant agreement CTQ201787773-P/AEI/FEDER. I.A. is grateful for support from the Alexander von Humboldt Foundation. We also acknowledge access to supercomputer resources as provided through grants from the Red Española de Supercomputación.

\section{References}


${ }^{1}$ Electric Field Effect in Atomically Thin Carbon Films, K. S. Novoselov, A. K. Geim, S. V. Morozov, D. Jiang, Y. Zhang, S. V. Dubonos, I. V. Grigorieva, A. A. Firsov, Science 306, 666-669 (2004).

${ }^{2}$ Semimetal-antiferromagnetic insulator transition in graphene induced by biaxial strain, S-H. Lee, S. Kim, K. Kim, Phys. Rev. B 86, 155436 (2012).

${ }^{3}$ Interaction-Driven Metal-Insulator Transition in Strained Graphene, H.-K. Tang, E. Laksono, J. N. B. Rodrigues, P. Sengupta, F. F. Assaad, S. Adam, Phys. Rev. Lett., 115, 186602 (2015)

${ }^{4}$ Correlation-Driven Dimerization and Topological Gap Opening in Isotropically Strained Graphene, S. Sorella, K. Seki, O. O. Brovko, T. Shirakawa, S. Miyakoshi, S. Yunoki, E. Tosatti, Phys. Rev. Lett. 121, 066402 (2018).

${ }^{5}$ Band Gap Opening by Two-Dimensional Manifestation of Peierls Instability in Graphene, S-H. Lee, H-J. Chung, J. Heo, H. Yang, J. Shin, U-I. Chung, S. Seo, ACS Nano, 5, 2964-2969 (2011).

${ }^{6}$ Correlated insulator behaviour at half-filling in magic-angle graphene superlattices, Y. Cao, V. Fatemi, A. Demir, S. Fang, S. L. Tomarken, J. Y. Luo, J. D. Sanchez-Yamagishi, K. Watanabe, T. Taniguchi, E. Kaxiras, R. C. Ashoori, P. Jarillo-Herrero, Nature, 556, 80-84 (2018).

${ }^{7}$ Tuning superconductivity in twisted bilayer graphene, M. Yankowitz, S. Chen, H. Polshyn, Y. Zhang, K. Watanabe, T. Taniguchi, D. Graf, A. F. Young, C. R. Dean, Science, 363, 1059-1064 (2019).

${ }^{8}$ Design of multi-functional 2D open-shell organic networks with mechanically controllable properties, I. Alcón, D. Reta, I. De P.R. Moreira, S. T. Bromley, Chem. Sci. 2017, 8, 1027.

${ }^{9}$ Existence of multi-radical and closed-shell semiconducting states in post-graphene organic Dirac materials, I. Alcón, F. Viñes, I de P. R. Moreira, S. T. Bromley, Nature Communications (2017), 8, 1957

${ }^{10}$ Toward Two-Dimensional $\pi$-Conjugated Covalent Organic Radical Frameworks, S. Wu, M. Li, H. Phan, D. Wang, T. S. Herng, J. Ding, Z. Lu, J. Wu, Angew. Chem. Int. Ed., 57, 8007 (2018).

${ }^{11}$ Antiferromagnetism in two-dimensional polyradical nanosheets, Y. Yang, C. Liu, X. Xu, Z. Meng, W. Tong, Z. Ma, C. Zhou, Y. Sun, Z. Sheng, Polym. Chem., 9, 5499-5503 (2018).

${ }^{12}$ Organic Radical-Linked Covalent Triazine Framework with Paramagnetic Behavior, Y. Jiang, I. Oh, S. Hun, J. Onur, B. Xiong, C. Sun, H. Lee, M. Huang, W. Kyung, S. Jin, H. Kim, J-U. Rohde, S. Kyu, K. J-W. Yoo, R. S. Ruoff, ACS Nano, 13, 5251 (2019).

${ }^{13}$ Kekulé valence bond order in an extended Hubbard model on the honeycomb lattice with possible applications to twisted bilayer graphene, X. Y. Xu, K. T. Law, P. A. Lee, Phys. Rev. B 98, 121406(R) (2018).

${ }^{14}$ Graphyne: Hexagonal network of carbon with versatile Dirac cones, B. G. Kim, H. J. Choi, Phys. Rev. B, 86, 115435 (2012).

${ }^{15}$ Dirac cone in $\alpha$-graphdiyne: a first-principles study, X. Niu, X. Mao, D. Yang, Z. Zhang, M. Si, D, Xue, Nanoscale Res. Lett. 8, 469 (2013).

${ }^{16}$ Kondo effect in a neutral and stable all organic radical single molecule break junction, R Frisenda, R. Gaudenzi, C. Franco, M. Mas-Torrent, C. Rovira, J. Veciana, I. Alcon, S. T. Bromley, E. Burzurí, H. S. J. van der Zant, Nano Lett. 15, 3109 (2015).

${ }^{17}$ Organic radicals on surfaces: towards molecular spintronics, M. Mas-Torrent,a N. Crivillers,a V. Mugnaini, I. Ratera, C. Rovira , J. Veciana , J. Mater. Chem., 19, 1691 (2009). 
${ }^{18}$ A robust molecular platform for non-volatile memory devices with optical and magnetic responses C. Simão, M. Mas-Torrent, N. Crivillers, V. Lloveras, J. M. Artés, P. Gorostiza, J. Veciana, C. Rovira, Nat. Chem. 3, 359 (2011).

${ }^{19}$ A nanoporous molecular magnet withreversible solvent-induced mechanical andmagnetic properties, D. Maspoch, D. Ruiz-Molina, K. Wurst, N. Domingo, M. Cavallini, F. Biscarini, J. Tejada, C. Rovira, J. Veciana, Nat. Mater. 2, 190 (2003).

${ }^{20}$ Structural control over spin localization in triarylmethyls ,I. Alcon and S. T. Bromley, RSC Adv., 5, 98593 (2015).

${ }^{21}$ Inhomogeneous electron gas, P. Hohenberg, W. Kohn, Phys. Rev. 136, B864 (1964).

${ }^{22}$ Effect of Fock exchange on the electronic structure and magnetic coupling in NiO, I. de P. R. Moreira, F. Illas, R. L. Martin, Phys. Rev. B 65, 155102 (2002).

${ }^{23}$ Hybrid exchange-correlation energy functionals for strongly correlated electrons: Applications to transitionmetal monoxides, F. Tran, P. Blaha, K. Schwarz, P. Novak, Phys. Rev. B 74, 155108 (2006).

${ }^{24}$ Electronic structure of single-layered undoped cuprates from hybrid density functional theory P. Rivero, I. de P.R. Moreira, F. Illas, Phys. Rev. B 81, 205123 (2010).

${ }^{25}$ Validation of the B3LYP/N07D and PBEO/N07D Computational Models for the Calculation of Electronic gTensors, V. Barone, P. Cimino, J. Chem. Theory Comput. 5 192-199 (2009).

${ }^{26}$ The Triplet-Singlet Gap in the m-Xylylene Radical: A Not So Simple One, D. Reta-Mañeru, A. K. Pal, I. de P. R. Moreira, S. N. Datta, F. Illas, J. Chem. Theory Comput., 10, 335-345 (2014).

${ }^{27}$ Quantum Chemical Approaches for Controlling and Evaluating Intramolecular Magnetic Interactions in Organic Diradicals, Daeheum Cho, Kyoung Chul Ko, Jin Yong Lee, Int. J. Quantum Chem. 116, 578-597 (2016).

${ }^{28}$ Magnetic Coupling Constants in Three Electrons Three Centers Problems from Effective Hamiltonian Theory and Validation of Broken Symmetry-Based Approaches, Daniel Reta, Ibério de P. R. Moreira, Francesc Illas, J. Chem. Theory Comput., 12, 3228-3235 (2016).

${ }^{29}$ Toward reliable density functional methods without adjustable parameters: The PBEO model, C. Adamo and V. Barone, J. Chem. Phys., 110, 6158 (1999).

${ }^{30}$ Semi-Metal-Insulator Transition of the Hubbard Model in the Honeycomb Lattice, S. Sorella, E. Tosatti, Europhys. Lett., 19, 699 (1992).

${ }^{31}$ Pinning the Order: The Nature of Quantum Criticality in the Hubbard Model on Honeycomb Lattice, F. F. Assaad, I. F. Herbut, Phys. Rev. X, 3, 031010 (2013).

32 Universal Quantum Criticality in the Metal-Insulator Transition of Two-Dimensional Interacting Dirac Electrons Y. Otsuka, S. Yunoki, and S. Sorella, Phys. Rev. X, 6, 011029 (2016).

${ }^{33}$ The role of electron-electron interactions in two-dimensional Dirac fermions, H-K. Tang, J. N. Leaw, J. N. B. Rodrigues, I. F. Herbut, P. Sengupta, F. F. Assaad, S. Adam, Science, 361, 570-574 (2018).

${ }^{34}$ Designer Dirac fermions and topological phases in molecular graphene, K. K. Gomes, W. Mar, W. Ko, F. Guinea, H. C. Manoharan, Nature 483, 306 (2012). 
${ }^{35}$ Pressure-Induced Conductivity in a Neutral Non-planar Spin-localized Radical, M. Souto, H. Cui, M. Peña-

Alvarez, V. G. Baonza, H. O. Jeschke, M. Tomic, R. Valenti, D. Blasi, I. Ratera, C. Rovira, J. Veciana, J. Am. Chem. Soc., $138,36,11517$ (2016).

${ }^{36}$ Emergence of an Antiferromagnetic Mott Insulating Phase in Hexagonal $\pi$-Conjugated Covalent Organic Frameworks, S. Thomas, H. Li, J-L. Bredas, Adv. Mater. 1900355 (2019).

37 Dynamic band-structure tuning of graphene moiré superlattices with pressure, M. Yankowitz, J. Jung, E. Laksono, N. Leconte, B. L. Chittari, K. Watanabe, T. Taniguchi, S. Adam, D. Graf, C. R. Dean, Nature, 404, 557 (2018).

${ }^{38}$ Tuning and Identification of Interband Transitions in Monolayer and Bilayer Molybdenum Disulfide Using Hydrostatic Pressure, X. Dou, K. Ding, D. Jiang, B. Sun, ACS Nano, 8, 7458 (2014).

39 Single Layer Molybdenum Disulfide under Direct Out-of-Plane Compression: Low-Stress Band-Gap Engineering, M. Peña-Álvarez, E. del Corro, Á. Morales-García, L. Kavan, M. Kalbac, O. Frank, Nano Lett. 15, 3139 (2015).

${ }^{40}$ [6]Cyclo-para-phenylmethine: An Analog of Benzene Showing Global Aromaticity and Open-Shell Diradical Character, Z. Li, T. Y. Gopalakrishna, Y. Ha, Yanwei Gu, L. Yuan, W. Zeng, D. Casanova, J. Wu, J. Am. Chem. Soc., 141, 16266 (2019).

${ }^{41}$ Strain-Induced Gap Modification in Black Phosphorus, A. S. Rodin, A. Carvalho, A. H. Castro Neto, Phys. Rev. Lett. 112, 176801 (2014).

${ }^{42}$ Structural phase transitions of phosphorene induced by applied strains, T. Hu, J. Dong, Phys. Rev. B, 92, 064114 (2015).

${ }^{43}$ Elastic coupling between layers in two-dimensional materials, Y. Gao, S. Kim, S. Zhou, H-C. Chiu, D. Nélias, C. Berger, W. de Heer, L. Polloni, R. Sordan, A. Bongiorno, E. Riedo, Nat. Mater 14, 714 (2015).

${ }^{44}$ Coarctate and Möbius: The Helical Orbitals of Allene and Other Cumulenes, M.H. Garner, R. Hoffmann, S. Rettrup, G.C. Solomon, ACS Cent. Sci., 4, 688 (2018).

${ }^{45}$ Molecular magnetism, J. Schnack (2004) In: Schollwöck U., Richter J., Farnell D.J.J., Bishop R.F. (eds)

Quantum Magnetism. Lecture Notes in Physics, vol 645. Springer, Berlin, Heidelberg (p. 155-194).

${ }^{46}$ New Platforms for Stable Carbon-Centered Radicals, K. Kato, A. Osuka, Angew. Chem. Int. Ed., 58, 8978 (2019).

${ }^{47} \mathrm{Ab}$ initio molecular simulations with numeric atom-centered orbitals, V. Blum et al. Comp. Phys. Comm. 180, 2175-2196 (2009).

${ }^{48}$ Accurate molecular van der Waals interactions from ground-state electron density and free-atom reference data, A. Tkatchenko, M. Scheffler, Phys. Rev. Lett. 102, 073005 (2009).

${ }^{49}$ Efficient integration for all-electron electronic structure calculation using numeric basis functions, V. Havu, V. Blum, P. Havu, M. J. Scheffler, Comput. Phys. 228, 8367 (2009).

${ }^{50}$ When Anatase Nanoparticles Become Bulklike: Properties of Realistic $\mathrm{TiO}_{2}$ Nanoparticles in the 1-6 nm Size Range from All Electron Relativistic Density Functional Theory Based Calculations, O. Lamiel-Garcia, K. C. Ko, J. Y. Lee, S. T. Bromley, F. J. Illas, Chem. Theory Comput. 13, 1785-1793 (2017). 
${ }^{51}$ Numeric atom-centered-orbital basis sets with valence-correlation consistency from $\mathrm{H}$ to Ar.I. Y. Zhang, X. Ren, P. Rinke, V. Blum, M. Scheffler, New J. Phys. 15, 123033 (2013).

${ }^{52}$ Magnetic properties and energy-mapping analysis, H. Xiang, C. Lee, H.-J. Koo, X. Gong, M.-H. Whangbo, Dalton Trans., 42, 823 (2013). 